\title{
Respiratory muscle training outcomes on swallowing biomechanics and respiratory function measures in normal subjects
}

João Rafael Sauzem Machado(1) Diego Fernando Dorneles Bilheri(1)

Lidia Lis Tomasi ${ }^{(1)}$

Eduardo Matias dos Santos Steid/(1)

Renata Mancopes ${ }^{(1)}$

(1) Universidade Federal de Santa Maria (UFSM), Santa Maria, RS, Brasil. Research support source: CAPES. Conflict of interests: Nonexistent

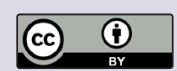

Received on: June 5, 2017

Accepted on: October 2, 2018

Corresponding address:

Renata Mancopes

Centro de Ciências da Saúde - UFSM

Av Roraima, n. 1000, Prédio 26, sala

1410, Bairro Camobi

CEP: 97105-900 - Santa Maria, Rio Grande do Sul, Brasil

E-mail: renata.mancopes@gmail.com

\section{ABSTRACT}

Purpose: to verify the outcomes of respiratory muscle training (RMT) with flow-oriented incentive spirometer on respiratory function measurements and their relationship with the swallowing biomechanics variables.

Methods: 29 subjects underwent training for seven consecutive days (three sets of ten repetitions for inspiration and expiration). The swallowing biomechanics was assessed by video fluoroscopy, using as temporal variable the pharyngeal transit time and as visuoperceptual variables the number of swallows, residues in pyriform sinuses and valleculae, and penetration/aspiration. Respiratory function measurements were assessed by maximal respiratory pressures and spirometry.

Results: RMT influenced pharyngeal transit time $(p=0.002)$ and maximal inspiratory pressure correlated with the number of swallows after RMT $(\rho=0.62, p=0.01)$. There was a significant increase in the maximum respiratory pressures post RMT $(p<0.0001)$.

Conclusion: RMT increased maximal respiratory pressures and influenced the reduction of pharyngeal transit time. Besides that, there was a relation between the maximum inspiratory pressure and the number of post-training swallows.

Keywords: Swallowing; Respiratory Exercises; Methods 


\section{INTRODUCTION}

Swallowing is divided into three phases: oral, pharyngeal and esophageal. The oral phase can be controlled voluntarily, whereas the pharyngeal and esophageal phases happen involuntarily. The pharyngeal phase, with its sequenced and coordinated actions, determines the flow of the swallowed material towards the esophagus, making it pass laterally to the laryngeal opening, without penetration of the swallowed contents towards the tracheobronchial tree ${ }^{1-3}$.

According to Li et al. ${ }^{4}$, the act of swallowing requires a series of voluntary and involuntary actions, highly coordinated with each other, so that the transportation of the food bolus along the pharynx happens safely. Therefore, there must be a deep biomechanical interaction from the moment the food bolus is propelled from the oral cavity towards the pharynx, through the pressure generated by the contact of the tongue with the palate, to the moment when the contractile structures responsible for the superior and anterior vertical displacement of the hyoid bone and the larynx protect the laryngeal opening that leads to the trachea. Steele et al. ${ }^{5}$ call hyolaryngeal complex the functional and anatomical integration of these structures of the pharyngeal phase.

Steele et al. ${ }^{6}$ published a literature review focused on elucidating and compiling scientific material on pathophysiological factors associated with swallowing dysfunctions. Among the factors pointed out are changes in lung capacity and strength, non-coordination between breathing / apnea / swallowing / respiration stages, reduction of the propulsion force of the bolus, increasing in the duration of the bolus stay in the pharyngeal phase of swallowing, changes in the hyoid bone mobility, and weakness of the suprahyoid muscles, which play a key role in generating the movement of the hyoid bone in the upper and anterior directions.

Respiratory muscular training (RMT) aims to improve respiratory performance through imposing a load on the respiratory system beyond its usual level of functioning, thus creating a training effect. Many RMT studies have been performed on healthy individuals, athletes and clinical populations with primary respiratory problems, always demonstrating, in the trained groups, absolute gains in lung capacity and lung strength variables ${ }^{7}$.

The use of respiratory stimulators aims to stimulate a deep inspiration, aiming at the maximum expansion of the lungs and the exercise of deep breathing. Respiron ${ }^{\circledR}$ is an incentive of nonlinear pressure load $^{8}$
(RI), used to RMT by forming a pressure threshold. It is responsible for increasing resistance to muscle fatigue and improving the respiratory function ${ }^{9}$.

Troche et al. $^{10}$ demonstrated the importance of RMT by using RI for the rehabilitation of dysphagia in parkinsonian subjects. There was an improvement in the swallowing function, attributed to the improvement of the hyolaryngeal complex function, resulting in a greater protection of the airways during swallowing.

In this context, this study objectives to verify the outcomes of RI assisted RMT on respiratory function measurements and their relationship with the swallowing biomechanics.

\section{METHODS}

A prospective longitudinal study was carried out, with approval of the Research Ethics Committee of the Federal University of Santa Maria, following Resolution 466/2012, under registration CAAE 23676813.8.0000.5346, and all participants signed a Consent Form agreeing to participate in the research.

The subjects were young adults, aged between 18 and 30 years, without previous diagnosis of respiratory disease, cold symptoms and / or respiratory conditions at the time of the evaluation, with no complaints of swallowing alterations and non-smokers. All subjects who presented any ventilatory alterations, evaluated by spirometry, were excluded from the study.

Thirty-two young people were evaluated at the start of the study, and two were excluded for presenting allergy to the barium contrast used in video fluoroscopy (VFD). Moreover, one was later excluded due to technical problems in the re-evaluation VFD recording, resulting in a sample of 29 subjects.

To analyze the biomechanics of swallowing, a VFD was performed using a $10 \mathrm{ml}$ scoop of paste consistency. Images were generated on a Siemens equipment, model Iconos R200, in fluoroscopy mode with 30 frames per second. The videos were recorded in the Zscan6 software, whose main technical characteristics are: images up to $720 \times 576$; 32Bit image resolution (32 million colors); 1440 dpi JPEG image format; video systems NTSC, PAL, SECAM (all standard); video up to 720x576 with real-time images (30 frames per second) AVI format and divX compressor, allowing recording on DVD or CD. During the VFD, subjects were evaluated in a sitting position with lateral projection. The video fluoroscopic image field included lips, oral cavity, cervical spine and proximal cervical esophagus. The mean dose value generated in this procedure was of 
$0.14 \mathrm{mR} /$ frame $(2.1 \mathrm{mR} / \mathrm{s})$. The dose measurements were performed under conditions that reproduce the technique and patient's positioning, using a $4 \mathrm{~cm}$ aluminum simulator and a Radcal electrometer, model 9010, with a specific ionization chamber for fluoroscopy procedures of $60 \mathrm{~cm}^{3}$.

Data collected through VFD were evaluated using temporal and visuoperceptual variables. The temporal variable analyzed was the pharyngeal transition time (PTT), expressed in seconds, and it considers as the beginning of the pharyngeal phase of swallowing the moment when the bolus was in the final region of the hard palate and the beginning of the soft palate, making an angle with the mandibular branch and the base of tongue, and as the end of the pharyngeal phase of swallowing the moment when the food bolus passed through the upper esophageal sphincter ${ }^{11}$.

The visuoperceptual variables were represented on numerical scales, as follows ${ }^{12}$ :

- Number of swallows (number of times the bolus is fragmented): 0 - 1 swallow; 1 - two swallows; 2 three swallows; 3 - four or more swallows.

- Residue in vallecula (stasis of the bolus in vallecula after complete swallowing): 0 - no stasis; 1 - residue filled up to $50 \%$ of the vallecula; 2 -residue filled more than $50 \%$ of the vallecula.

- Residue in pyriform sinus (food bolus stasis in pyriform sinuses after complete swallowing): 0 - no stasis; 1 - mild to moderate stasis; 2 - severe stasis, filling the pyriform sinuses.

- Penetration / Aspiration: 0 - normal; 1 - penetration; 2 - aspiration.

The variables under study were analyzed by two blinded referees, each one of them with five years of experience in VFD analysis. Referees received prior training in Movie Maker ${ }^{\circledR}$ software to perform video analysis for both visuoperceptual and temporal variables.

In order to evaluate the respiratory function measurements, a spirometry was performed. For the spirometry, the subject was in a sitting position with the spirometer disposable mouthpiece adapted to the mouth and was encouraged to perform a maximal inspiration followed by a rapid and sustained expiration of at least six seconds. A nasal clip was used to assist in the airway sealing. The measurements were performed with a MicroQuark spirometer, using OneFlow 3.0.0 software to evaluate the measurements.

Both the technique and the quality controls were performed according to the references described in the
Guidelines for Pulmonary Function Tests published by the Brazilian Society of Pulmonology and Tisiology ${ }^{13}$. The measured variables were: forced vital capacity (FVC), measured in liters, and the percentage over the predicted value was calculated; forced expiratory volume in the first second (FEV1), measured in liters per second and the percentage over the predicted value was calculated.

The maximum inspiratory pressure (MIP) and maximum expiratory pressure (MEP) measurements were performed with an MDV-500 Globalmed digital manovacuometer, which is graduated from - $500 /+$ $500 \mathrm{cmH}_{2} \mathrm{O}$. MIP and MEP measurements were taken with the subjects seated, as described by Black and Hyat $^{14}$. According to the SBPT ${ }^{13}$ guidelines, the subject was seated comfortably with a nasal clip, and all the instructions on the test were given prior to the evaluation. Up to six measurements of MIP and MEP were performed for each subject, with a one-minute interval between each repetition, to produce three acceptable maneuvers, without leaks and lasting at least two seconds each. The value adopted in the study as respiratory muscular strength of the individual was the greater value obtained, if there were at least two reproducible maneuvers with values that did not differ by more than $10 \%$ on the highest value.

The evaluation of the respiratory function measurements (spirometry and manovacuometry) were randomly distributed among subjects, in order to minimize the effort factor among the participants.

The training with respiratory exercise was performed at home, with previous orientations given by a physiotherapist, through flow RI on the Respiron $®$, for a period of seven consecutive days. For inspiratory training, all participants should perform daily three sets of ten repetitions, starting from the Functional Residual Capacity (FRC) until reaching Inspiratory Capacity (IC) and sustaining the spheres for a period of five to eight seconds ${ }^{15}$. For the expiratory training, the Respiron ${ }^{\circledR}$ use was reversed. Each participant was instructed to, during a week, daily perform three sets of ten repetitions, starting from IC until FRC, with a one-minute rest between each series. Daily contacts were made with each participant in order to reinforce the importance of conducting the training ${ }^{16}$.

Data collected were analyzed using the Statistical Package for Social Science (SPSS) version 17. Wilcoxon and t-test were performed to verify the significance between the categorical variables. For the non-categorical variables, a Test for Equality of Two 
Proportions was used. Also, the Spearman test was performed to correlate the biomechanics of deglutition with the respiratory function measurements.

To determine the agreement between referees, the Wilcoxon test was applied for the temporal variable and the Kappa test for the visuoperceptual ones. The degree of agreement was defined according to Landis and Koch ${ }^{17}$ : $<0.00$ poor agreement; $0.00-0.19$ poor agreement; $0.20-0.39$ weak agreement; 0.40 - 0.59 moderate agreement; $0.60-0.79$ substantial agreement; $0.80-1.00$ almost perfect agreement. It was found that for the PTT variable the referees' agreement was of $100 \%(p=1.00)$, which indicates almost perfect agreement, as well as for the visuoperceptual variables $($ Kappa $=1.0, p<0.001)$.

The results were expressed as mean \pm standard deviation and percentage, considering statistically $p$ values $<0.05$.

\section{RESULTS}

The sample consisted of 29 young adults, aged 20.9 \pm 3.2 years, $75 \%$ females. Table 1 presents the results regarding respiratory function, showing that all subjects were healthy at the time of evaluation.

Table 1. Respiratory function of the evaluated subjects

\begin{tabular}{cc}
\hline Variables & Mean \pm SD \\
\hline $\mathrm{FEV}_{1}(\mathrm{l})$ & $3.5 \pm 0.5$ \\
$\% \mathrm{FEV}$ & $94.5 \pm 9.9$ \\
$\mathrm{FVC}(\mathrm{I})$ & $4.7 \pm 1.0$ \\
$\% \mathrm{FVC}$ & $107.6 \pm 10.6$ \\
$\mathrm{FEV}_{1} / \mathrm{FVC}(\%)$ & $87.32 \pm 7.5$ \\
\hline
\end{tabular}

$\mathrm{FEV}_{1}$ - forced expiratory volume in the first second; \% FEV - percentage of predicted forced expiratory volume in the first second; FVC - forced vital capacity; \% FVC - percentage of predicted forced vital capacity; FEV $1 /$ FVC (\%) - Tiffeneau index.

The values for MIP and MEP pre and post respiratory exercise presented mean values of $79.3 \pm 27.8$ $X 101 \pm 32.8$ and $77.9 \pm 29.3 \times 95.2 \pm 35.3 \mathrm{cmH}_{2} \mathrm{O}$, respectively.

Table 2 presents the values of the swallowing biomechanics variables analyzed before and after respiratory muscle exercise.

Table 2. Swallowing biomechanics variables pre and post respiratory muscle exercise

\begin{tabular}{|c|c|c|c|c|c|}
\hline \multirow{2}{*}{\multicolumn{2}{|c|}{ Variables }} & & \multicolumn{2}{|c|}{ Respiratory exercise } & \multirow{2}{*}{ p-value } \\
\hline & & & Pre & Post & \\
\hline \multicolumn{6}{|l|}{ Temporal } \\
\hline & PTT (s) & & $0.7 \pm 0.3$ & $0.6 \pm 0.3$ & $0.002^{*}$ \\
\hline \multicolumn{6}{|c|}{ Visuoperceptuals } \\
\hline & Number of swallows & & & & \\
\hline & & $1(0)$ & $68.8 \%$ & $81.3 \%$ & \\
\hline & & 2(1) & $25 \%$ & $18.7 \%$ & $0.20^{\star *}$ \\
\hline & & $3(2)$ & $6.3 \%$ & - & \\
\hline & Post-deglutition VL residue & & & & \\
\hline & & 1 & $15.6 \%$ & $15.6 \%$ & $100 * *$ \\
\hline & & 0 & $84.4 \%$ & $84.4 \%$ & $1.00^{\times x}$ \\
\hline & Post-deglutition PS residue & & & & \\
\hline & & 1 & $6.2 \%$ & 3.1 & \\
\hline & & 0 & $93.8 \%$ & $96.9 \%$ & $0.50^{\star *}$ \\
\hline & $\mathrm{P} / \mathrm{A}$ & & & & \\
\hline & & 2 & - & - & \\
\hline & & 1 & - & - & $1.00 * *$ \\
\hline & & 0 & $100 \%$ & $100 \%$ & \\
\hline
\end{tabular}

PTT - pharyngeal transition time; $s$ - seconds; VL - valleculae; PS - pyriform sinus; P/A - penetration / aspiration.

* Wilcoxon Test

** Two proportions test. 
Table 2 shows that, when compared pre and post respiratory exercise, only the PTT variable presented a statistically significant difference $(p=0.002)$.

Regarding the correlation between respiratory variables and swallowing biomechanics variables, the only correlation observed was between the post-exercise MIP and the post-exercise number of swallows $(\rho=0.62, p=0.01)$. The other variables did not present correlations.

The effects of respiratory exercise with Respiron ${ }^{\circledR}$ on respiratory function showed only significant difference on maximal respiratory pressures (Table 3 ).

Table 3. Comparison of respiratory variables pre and post respiratory muscle exercise

\begin{tabular}{cccc}
\hline \multirow{2}{*}{ Variables } & \multicolumn{2}{c}{ Respiratory exercise } & \multirow{2}{*}{ p-value* } \\
\cline { 2 - 3 } & Pre & Post & $<0.0001$ \\
MIP & $79.3 \pm 27.8$ & $101 \pm 32.8$ & $<0.0001$ \\
MEP & $77.9 \pm 29.3$ & $95.2 \pm 35.5$ & 0.70 \\
FEP & $350.6 \pm 120.9$ & $363 \pm 78.2$ & 0.50 \\
$\%$ FEP & $70 \pm 20.6$ & $73.4 \pm 14.2$ & 0.40 \\
FEV & $3.5 \pm 0.5$ & $3.6 \pm 0.5$ & 0.30 \\
FEV $_{1}$ & $94.5 \pm 9.9$ & $96.8 \pm 10$ & 0.30 \\
FVC & $4.7 \pm 1.0$ & $4.5 \pm 0.9$ & 0.50 \\
\%FV & $107.6 \pm 10.6$ & $104.1 \pm 20.2$ & \\
\hline
\end{tabular}

MIP - maximum inspiratory pressure; MEP - maximum expiratory pressure; FEP - forced expiratory peak; \% FEP - percentage over predicted forced expiratory peak; $\mathrm{FEV}_{1}$ - forced expiratory volume in the first second; \% $\mathrm{FEV}_{1}$ - percentage over predicted forced expiratory volume in the first second; FVC - forced vital capacity;

$\%$ FVC - percentage over predicted forced vital capacity.

* Student's t-test

\section{DISCUSSION}

Muscle fibers acquire the ability to change their physiological and biochemical properties according to muscle training, leading to an increase in the amount of fiber internal proteins responsible for the capacity of contraction and force generation. This induces these cells to an adaptation and consequent increase of their mechanical performance. For Guyton and Hall ${ }^{18}$, this means muscle plasticity and the ability to react to loads imposed by various forms of muscular exercise. As a result, an improvement in contraction power is obtained, which is determined not only by the force exerted by the muscle, but also by the contraction velocity.

In the present study, only the PTT variable presented a significant difference between pre and post RMT ( $p$ $=0.002$ ). However, this variable did not correlate with any respiratory variable analyzed. Thus, it could be inferred that PTT depends on other factors, in addition to respiratory measures, such as the sensitivity of the pharyngeal region, due to the increase in the airflow caused by the IR, and measures of activation of the pharyngeal musculature, which should be better investigated.
Studies that verified the impact of using Respiron ${ }^{\circledR}$ on the biomechanics of swallowing have not been found in the literature to date. Fregosi and Ludlow ${ }^{19}$ stated that muscular coordination in the pharyngeal path is of fundamental importance for swallowing and breathing to occur in an effective and safe way, being necessary for this, among other factors, muscular power. In the present study, there was a reduction in the number of swallows after RMT and this was correlated with MIP, which presented a significant increase after the training.

Reyes et al. $^{20}$, in a randomized controlled study, proposed RMT (inspiratory and expiratory) for individuals with Huntington's disease. The trained group achieved a significant improvement compared to the control group in variables such as maximal pressures (inspiratory and expiratory), forced expiratory peak and deglutition, and in a quality of life questionnaire on swallowing, in 2 and 4-month RMT assessments. In this sense, it is possible to infer that RMT potentiated the musculature responsible for swallowing, inducing reduction in the number of swallows and greater tonic protection of the airways.

Rosa's et al. ${ }^{16}$ study corroborates the findings obtained in this study. The authors analyzed the use of 
the inverted Respiron ${ }^{\circledR}$ on respiratory muscle strength in young adults (aged 20-30), showing an increase of 15 $\mathrm{cmH}_{2} \mathrm{O}$ in MIP and $23 \mathrm{cmH}_{2} \mathrm{O}$ in MEP, after a two-month training, with triweekly sessions of 10 repetitions with a one-minute interval between each maneuver.

Another study used linear load RI for expiratory training (EMST-15) in a sample of stroke victims. Measurements of lung strength and lung capacity were performed at baseline, at four weeks and at 12 weeks post-training. Results showed a considerable gain in pulmonary strength and lung capacity variables, as well as in the capacity to generate subglottic pressure for coughing, causing a reduced incidence of aspiration pneumonia in secondary observations ${ }^{7}$.

Regarding the effects of RMT with flow RI, in this study a significant difference in respiratory pressures $(p$ $<0.0001$ ) was found, which was expected. Respiron ${ }^{\circledR}$ is commonly indicated by physiotherapists to promote tidal volume increase and, thus, improve pulmonary expansion in individuals affected by conditions of different etiologies ${ }^{16}$. In the literature, the main focus of RI studies has been the pulmonary expansion. Only in the last 15 years $^{21}$ it has been presented as a resource for exercising the respiratory muscles.

Among the limitations of the present study are pointed the reduced sample size, the scarcity of other studies that evaluate the effects of Respiron ${ }^{\circledR}$ on swallowing in normal subjects and lack of an objective analysis of the other variables of the swallowing biomechanics.

\section{CONCLUSION}

Although the effects of respiratory muscle exercise with the use of Rl are already known in pulmonary force and function, no studies were found to indicate their impact on the swallowing biomechanics.

This study shows that RMT has an effect on maximum respiratory pressures, a result already pointed out in the literature. As for RMT effects on the biomechanics of swallowing, there is a correlation between the MIP and the number of swallows posttraining, which may be related to a reduction in PTT.

Thus, new studies addressing this subject, including a larger number of subjects, evaluation of the sensitivity of the anatomical regions responsible for swallowing and relations with the movement / displacement of the hyoid bone, are necessary.

\section{REFERENCES}

1. Costa M. Deglutição \& Disfagia: bases morfofuncionais e videofluoroscópicas. Rio de Janeiro: Med Book; 2013.

2. Chaves RD, Carvalho CRF, Cukier A, Stelmach R, Andrade CRF. Sintomas indicativos de disfagia em portadores de DPOC. J Bras Pneumol. 2011;37(2):176-83.

3. Dozier TS, Brodsky MB, Michel Y, Walters BC, Martin-Harris B. Coordination of swallowing and respiration in normal sequential cup swallows. Laryngoscope. 2006;116(8):1489-93.

4. Li Q, Minagi $Y$, Hori K, Kondoh J, Fujiwara S, Tamine $\mathrm{K}$ et al. Coordination in oro-pharyngeal biomechanics during human swallowing. Physiology \& Behavior. 2015;147:300-5.

5. Steele CM, Miller AJ. Sensory input pathways and mechanisms in swallowing: a review. Dysphagia. 2010;25(4):323-33.

6. Steele CM, Cichero JAY. Physiological factors related to aspiration risk: a systematic review. Dysphagia. 2014;29(3):295-304.

7. Kulnik ST, Rafferty GF, Birring SS, Moxham J, Kalra L. A pilot study of respiratory muscle training improve cough effectiveness and reduce the incidence of pneumonia in acute stroke: study protocol for a randomized controlled trial. Trials. 2014; 15:123.

8. Sarmento G. Recursos em fisioterapia cardiorrespiratória. São Paulo: Manole; 2012.

9. Paiva DN, Asmann LB, Bordin DF, Gass R, Jost RT, Bernardo-Filho $M$ et al. Inspiratory muscle training with threshold or incentive spirometry: wich is the most effective? Rev Port Pneumol. 2015;21(2):76-81.

10. Troche MS, Okaun MS, Rosenbek JC, Musson N, Fernandez $\mathrm{HH}$, Rodriquez $\mathrm{R}$ et al. Aspiration and swallowing in Parkinson disease and rehabilitation with EMST. Neurology. 2010;75(21):1912-9.

11. Kendall KA, Leonard RJ, McKenzie SW. Accommodation to changes in bolus viscosity in normal deglutition: a videofluoroscopic study. Ann Otol Rhinol Laryngol. 2001;110(11):1059-65.

12. Baijens LWJ, Speyer R, Passos VL, Pilz W, Roodengurg N, Clave P. Swallowing in Parkinson patients versus healthy controls: reliability of measurements in videofluoroscopy. Gastroenterology Research and Practice. 2011; Volume 2011, Article ID 380682, 9 pages. http:// dx.doi.org/10.1155/2011/380682 
13. Pereira CAC. Espirometria. J Pneumol. 2002;28(Supl 3):S1-S82.

14. Black LF, Hyatt RE. Maximal respiratory pressures: normal values and relationship to age and sex. Am Rev Respir Dis. 1969;99(5):696-702.

15. Ysayama L, Lopes LR, Silva AMO, Andreollo NA. A influência do treinamento muscular respiratório pré-operatório na recuperação de pacientes submetidos à esofagectomia. Arq Bras Cir Dig. 2008;21(2):61-4.

16. Rosa R, Santos GK, Siqueira AB, Toneloto MGC. Inspirômetro de incentivo invertido como exercitador da musculatura respiratória em indivíduos saudáveis. Rev Intellectus. 2013.25:177-97.

17. Landis JR, Koch GG. The Measurement of observer agreement for categorical data. Biometrics. 1977;33(1):159-74.

18. Guyton AC, Hall JE. Tratado de fisiologia médica. 10ㅇe ed. Rio de Janeiro: Guanabara Koogan; 2002.

19. Fregosi RF, Ludlow CL. Activation of upper airway muscles during breathing and swallowing. J Appl Physiol. 2014;116(3):291-301.

20. Reyes A, Cruickshank T, Nosaka K, Ziman M. Respiratory muscle training on pulmonary and swallowing function in patients with huntington's disease: a pilot randomised controlled trial. Clinical Rehabilitation. 2014; PII: 0269215514564087.

21. Scalan CL, Wilkins RL, Stoller JK. Fundamentos da terapia respiratória de Egan. 7으. ed. São Paulo: Manole; 2000. 